DESY 01-213

hep-ph/0112254

\title{
FUNDAMENTAL PHYSICS AT AN X-RAY FREE ELECTRON LASER
}

\author{
A. RINGWALD \\ Deutsches Elektronen-Synchrotron DESY, Notkestraße 85, \\ D-22607 Hamburg, Germany
}

\begin{abstract}
X-ray free electron lasers (FELs) have been proposed to be constructed both at SLAC in the form of the so-called Linac Coherent Light Source as well as at DESY, where the socalled XFEL laboratory is part of the design of the electron-positron linear collider TESLA. In addition to the immediate applications in condensed matter physics, chemistry, material science, and structural biology, X-ray FELs may be employed also to study some physics issues of fundamental nature. In this context, one may mention the boiling of the vacuum (Schwinger pair creation in an external field), horizon physics (Unruh effect), and axion production. We review these X-ray FEL opportunities of fundamental physics and discuss the necessary technological improvements in order to achieve these goals.
\end{abstract}

\section{Introduction}

There are definite plans for the construction of free electron lasers (FELs) in the X-ray band, both at the Standord Linear Accelerator Center (SLAC), where the so-called Linac Coherent Light Source (LCLS) has been proposed 1 , as well as at DESY, where the so-called XFEL laboratory is part of the design of the electron-positron $\left(e^{+} e^{-}\right)$linear collider TESLA (TeVEnergy Superconducting Linear Accelerator) 6 .

X-ray free electron lasers will give us new insights into natural and life sciences. X-rays play a crucial role when the structural and electronic properties of matter are to be studied on an atomic scale. The spectral characteristics of the planned X-ray FELs, with their high power, short pulse length, narrow bandwidth, spatial coherence, and a tunable wavelength, make them ideally suited for applications in atomic and molecular physics, plasma physics, condensed matter physics, material science, chemistry, and structural biology 1.2 .3 .

In addition to these immediate applications, X-ray FELs may be employed also to study some physicsissues of fundamental nature 6 . In this context, one may mention the boiling of the vacuum 0 6.6 (Schwinger pair creation in an external field), horizon physics 10,11 (Unruh effect), and axion production 12 , 13. In this contribution, I shall concentrate on these particle physics opportunities of X-ray FELs. I shall also discuss the necessary improvements in X-ray FEL technology in order to achieve these goals.

\section{X-Ray Free Electron Lasers}

Before we enter the discussion of particle physics issues, let us briefly review the principle of $\mathrm{X}$-ray free electron lasers.

\footnotetext{
${ }^{a}$ Invited talk at the Workshop on Electromagnetic Probes of Fundamental Physics, Erice, Italy, October 2001.
} 


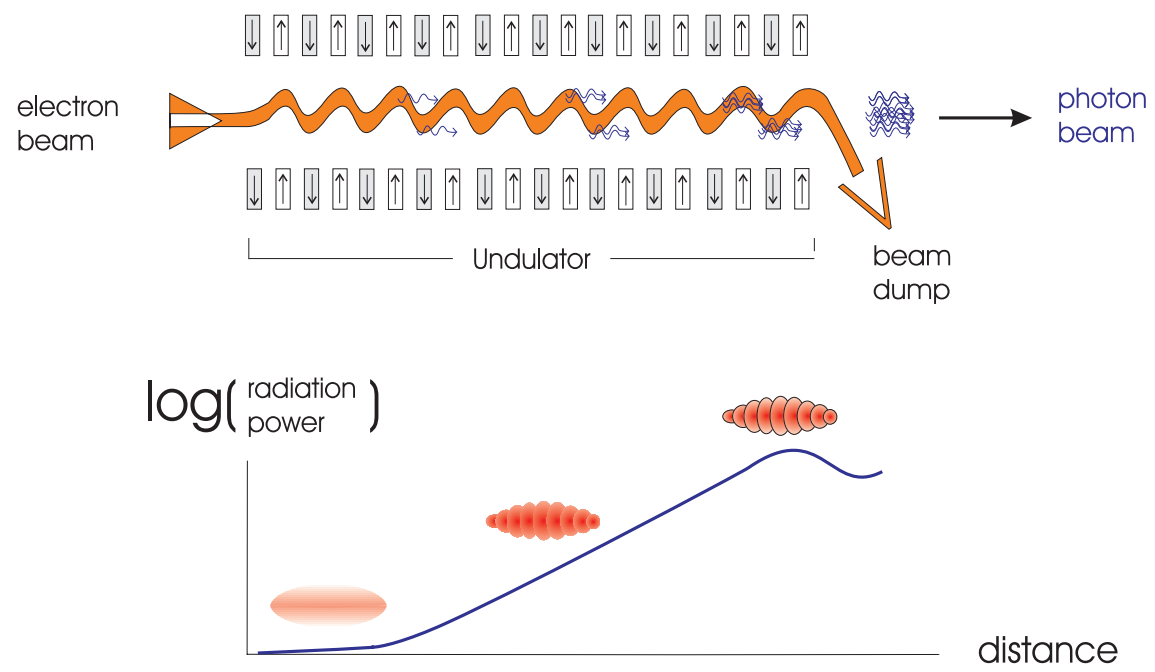

Figure 1: Principle of a single-pass X-ray free electron laser in the self amplified spontaneous emission mode 3.

Conventional lasers yield radiation typically in the optical band. The reason is that in these devices the gain comes from stimulated emission from electrons bound to atoms, either in a crystal, liquid dye, or a gas. The amplification medium of free electron lasers 14 , on the other hand, is free (unbounded) electrons in bunches accelerated to relativistic velocities with a characteristic longitudinal charge density modulation (cf. Fig. 1). The radiation emitted by an FEL can be tuned over a wide range of wavelengths, which is a very important advantage over conventional lasers.

The basic principle of a single-pass free electron laser operating in the self amplified spontaneous emission (SASE) mode15 is as follows. It functions by passing an electron beam pulse of energy $E_{e}$ of small cross section and high peak current through a long periodic magnetic

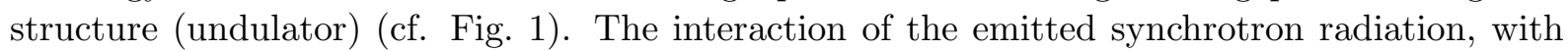
opening angle

$$
1 / \gamma=m_{e} c^{2} / E_{e}=2 \cdot 10^{-5}\left(25 \mathrm{GeV} / E_{e}\right)
$$

where $m_{e}$ is the electron mass, with the electron beam pulse within the undulator leads to the buildup of a longitudinal charge density modulation (micro bunching), if a resonance condition,

$$
\lambda=\frac{\lambda_{\mathrm{U}}}{2 \gamma^{2}}\left(1+\frac{K_{\mathrm{U}}^{2}}{2}\right)=0.3 \mathrm{~nm}\left(\frac{\lambda_{\mathrm{U}}}{1 \mathrm{~m}}\right)\left(\frac{1 / \gamma}{2 \cdot 10^{-5}}\right)^{2}\left(\frac{1+K_{\mathrm{U}}^{2} / 2}{3 / 2}\right),
$$

is met. Here, $\lambda$ is the wavelength of the emitted radiation, $\lambda_{\mathrm{U}}$ is the length of the magnetic period of the undulator, and $K_{\mathrm{U}}$ is the undulator parameter,

$$
K_{\mathrm{U}}=\frac{e \lambda_{\mathrm{U}} B_{\mathrm{U}}}{2 \pi m_{e} c}
$$

which gives the ratio between the average deflection angle of the electrons in the undulator magnetic field $B_{\mathrm{U}}$ from the forward direction and the typical opening cone of the synchrotron radiation. The undulator parameter should be of order one on resonance. The electrons in the developing micro bunches eventually radiate coherently - the gain in radiation power $P$,

$$
P \propto e^{2} N_{e}^{2} B_{\mathrm{U}}^{2} \gamma^{2}
$$

over the one from incoherent spontaneous synchrotron radiation $\left(P \propto N_{e}\right)$ being proportional to the number $N_{e} \geq 10^{9}$ of electrons in a bunch (cf. Fig. 目 (left)) - and the number of emitted 


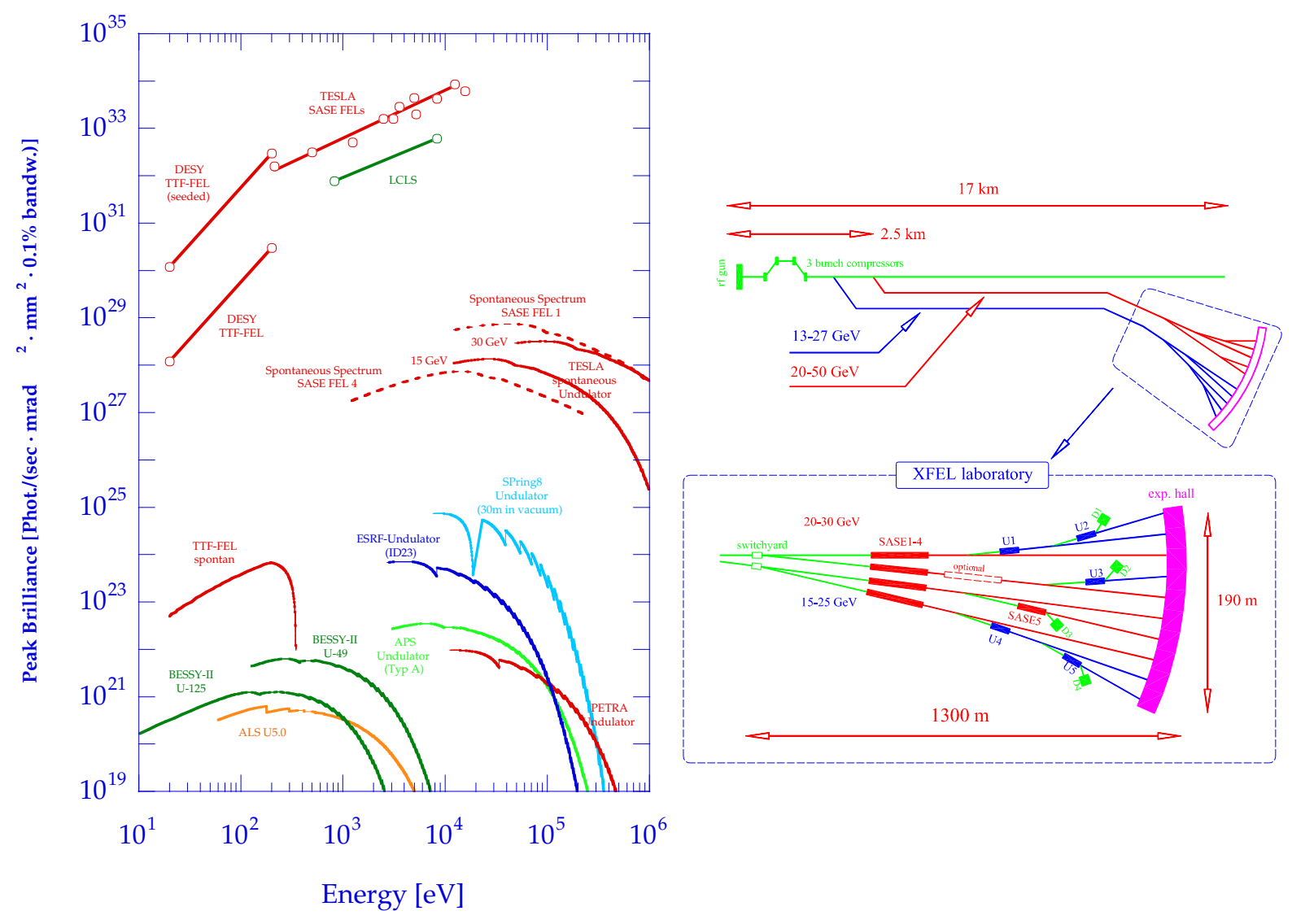

Figure 2: Left: Spectral peak brilliance of X-ray FELs and undutators for spontaneous radiation at TESLA, together with that of third generation synchrotron radiation sources 3 . For comparison, the spontaneous spectrum of an X-ray FEL undulator is shown Right: Schematic view of the TESLA XFEL electron beam transport (top) and the XFEL laboratory (bottom) 3 .

photons grows exponentially until saturation is reached. The radiation has a high power, short pulse length, narrow bandwidth, is fully polarized, transversely coherent, and has a tunable wavelength.

The concept of using a high energy electron linear accelerator for building an X-ray FEL was first proposed for the Stanford Linear Accelerator 1 . The feasability of a single-pass FEL operating in the SASE mode has recently been demonstrated 16 down to a wavelength of $80 \mathrm{~nm}$ using electron bunches of high charge density and low emittance from the linear accelerator at the TESLA test facility (TTF) at DESY. An X-ray FEL laboratory is planned as an integral part of TESLA A. B (cf. Fig. 目 (right)). Some characteristics of the radiation from the planned X-ray FELs at TESLA are listed in Table 1 .

Table 1: Typical photon beam properties of the SASE FELs at TESLA 3.

\begin{tabular}{|lc||c|c|c|c|c|}
\hline & unit & SASE 1 & SASE 2 & SASE 3 & SASE 4 & SASE 5 \\
\hline wavelength & $\mathrm{nm}$ & $0.1 \div 0.5$ & $0.085 \div 0.133$ & $0.1 \div 0.24$ & $0.1 \div 1.0$ & $0.4 \div 5.8$ \\
peak power & $\mathrm{GW}$ & 37 & 19 & 22 & 30 & $110 \div 200$ \\
average power & $\mathrm{W}$ & 210 & 110 & 125 & 170 & $610 \div 1100$ \\
numb. photons per pulse & $\#$ & $1.8 \cdot 10^{12}$ & $8.2 \cdot 10^{11}$ & $1.1 \cdot 10^{12}$ & $1.5 \cdot 10^{12}$ & $2.2 \div 58 \cdot 10^{13}$ \\
bandwidth (FWHM) & $\%$ & 0.08 & 0.07 & 0.08 & 0.08 & $0.29 \div 0.7$ \\
pulse duration (FWHM) & $\mathrm{fS}$ & 100 & 100 & 100 & 100 & 100 \\
\hline
\end{tabular}




\section{Applications in Particle Physics}

The spectral characteristics of X-ray free electron lasers suggest immediate applications in condensed matter physics, chemistry, material science, and structural biology, which are reviewed in the conceptual 12 and technical 3 design reports of the planned X-ray FEL facilities. In this section, we want to emphasize that X-ray FELs may be employed also to study some particle physics issues. In this context, one may mention the boiling of the vacuum 0.60 , 8 (Schwinger pair creation in an external field), horizon physics 10,11 (Unruh effect), and axion production 12.13.

Whereas the last application mainly requires large average radiation power $\langle P\rangle$, the first two applications require very large electric fields and thus high peak power densities $P /\left(\pi \sigma^{2}\right)$, where $\sigma$ is the laser spot radius. Here, one could make use of the possibility to focus the X-ray beam to a spot of small radius, hopefully down to the diffraction limit, $\sigma \gtrsim \lambda \simeq \mathcal{O}(0.1) \mathrm{nm}$. In this way, one may obtain very large electric fields and accelerations,

$$
\begin{aligned}
\mathcal{E} & =\sqrt{\mu_{0} c \frac{P}{\pi \sigma^{2}}}=1.1 \cdot 10^{17} \frac{\mathrm{V}}{\mathrm{m}}\left(\frac{P}{1 \mathrm{TW}}\right)^{1 / 2}\left(\frac{0.1 \mathrm{~nm}}{\sigma}\right) \\
a & =\frac{e \mathcal{E}}{m_{e}}=1.9 \cdot 10^{28} \frac{\mathrm{m}}{\mathrm{s}^{2}}\left(\frac{P}{1 \mathrm{TW}}\right)^{1 / 2}\left(\frac{0.1 \mathrm{~nm}}{\sigma}\right)
\end{aligned}
$$

much larger than those obtainable with any optical laser of the same power.

\subsection{Boiling the Vacuum}

Spontaneous particle creation from vacuum induced by an external field, first put forth to examine the production of $e^{+} e^{-}$pairs in a static, spatially uniform electric field 17 and often referred to as the Schwinger mechanism, ranks among the most intriguing nonlinear phenomena in quantum field theory. Its consideration is theoretically important, since it requires one to go beyond perturbation theory, and its experimental observation would verify the validity of the theory in the domain of strong fields. Moreover, this mechanism has been applied to many problems in contemporary physics. ranging from black hole quantum evaporation 18 to particle production in hadronic collisions 19 and in the early universe 20, to mention only a few. One may consult the monographs 21 for a review of further applications, concrete calculations and a detailed bibliography.

It is known since a long time that in the background of a static, spatially uniform electric field the vacuum in quantum electrodynamics (QED) is unstable and, in principle, sparks with spontaneous emission of $e^{+} e^{-}$pairs 17 . However, a sizeable rate for spontaneous pair production requires extraordinary strong electric field strengths $\mathcal{E}$ of order or above the critical value

$$
\mathcal{E}_{c} \equiv \frac{m_{e} c^{2}}{e \lambda_{e}}=\frac{m_{e}^{2} c^{3}}{e \hbar} \simeq 1.3 \times 10^{18} \mathrm{~V} / \mathrm{m}
$$

Otherwise, for $\mathcal{E} \ll \mathcal{E}_{c}$, the work of the field on a unit charge $e$ over the Compton wavelength of the electron $\lambda_{e}=\hbar /\left(m_{e} c\right)$ is much smaller than the rest energy $2 m_{e} c^{2}$ of the produced $e^{+} e^{-}$ pair, the process can occur only via quantum tunneling, and its rate is exponentially suppressed,

$$
\frac{\mathrm{d}^{4} n_{e^{+} e^{-}}}{\mathrm{d}^{3} x \mathrm{~d} t} \sim \frac{c}{4 \pi^{3} \lambda_{e}{ }^{4}} \exp \left[-\pi \frac{\mathcal{E}_{c}}{\mathcal{E}}\right]
$$

Unfortunately, it seems inconceivable to produce macroscopic static fields with electric field strengths of the order of the Schwinger critical field (7) in the laboratory. In view of this 
Table 2: Faser parameters and derived quantities relevant for estimates of the rate of spontaneous $e^{+} e^{-}$pair production 1. The colmmn labeled "Optical" lists parameters which are typical for a petawatt-class $(1 \mathrm{PW}=$ $10^{15} \mathrm{~W}$ ) optical laser 35 , focused to the diffraction limit, $\sigma=\mathrm{f}$. The column labeled "Design" displays desigen parameters of the planned XFEL at DESY ("SASE-5" in Ref. 3 and Table 11). Similar values apply for LCLSL. The column lapeled "Focus: Available" shows typical values which can be achieved with present day methods of X-ray focusing 36.37: It assumes that the XFEL X-ray beam can be focused to a rms spot radius of $\sigma \simeq 21 \mathrm{~nm}$ with an energy extraction efficiency of $1 \%$ 36. The column labeled "Focus: Goal" shows parameters which are theoretically possible by increasing the energy extraction of LCLS (by the tapered undulator technique) and by a yet unspecified method of diffraction-limited focusing of X-rays $\mathrm{G}$.

\begin{tabular}{|c|c|c|c|c|c|}
\hline \multicolumn{6}{|c|}{ Laser Parameters } \\
\hline & & Optical 35 & \multicolumn{3}{|c|}{ X-ray FEL } \\
\hline & & $\begin{array}{c}\text { Focus: } \\
\text { Diffraction limit }\end{array}$ & Design 3 & $\begin{array}{c}\text { Focus: } \\
\text { Available } 36\end{array}$ & $\begin{array}{l}\text { Focup } \\
\text { Goal } 6\end{array}$ \\
\hline Wavelength & $\lambda$ & $1 \mu \mathrm{m}$ & $0.4 \mathrm{~nm}$ & $0.4 \mathrm{~nm}$ & $0.15 \mathrm{~nm}$ \\
\hline Photon energy & $\hbar \omega=\frac{h c}{\lambda}$ & $1.2 \mathrm{eV}$ & $3.1 \mathrm{keV}$ & $3.1 \mathrm{keV}$ & $8.3 \mathrm{keV}$ \\
\hline Peak power & $P \quad{ }^{\lambda}$ & $1 \mathrm{PW}$ & $110 \mathrm{GW}$ & $1.1 \mathrm{GW}$ & 5 TW \\
\hline Spot radius (rms) & $\sigma$ & $1 \mu \mathrm{m}$ & $26 \mu \mathrm{m}$ & $21 \mathrm{~nm}$ & $0.15 \mathrm{~nm}$ \\
\hline Coherent spike length (rms) & $\triangle t$ & $500 \mathrm{fs} \div 20 \mathrm{ps}$ & $0.04 \mathrm{fs}$ & $0.04 \mathrm{fs}$ & $0.08 \mathrm{ps}$ \\
\hline \multicolumn{6}{|c|}{ Derived Quantities } \\
\hline Peak power density & $S=\frac{P}{\pi \sigma^{2}}$ & $3 \times 10^{26} \frac{\mathrm{W}}{\mathrm{m}^{2}}$ & $5 \times 10^{19} \frac{\mathrm{W}}{\mathrm{m}^{2}}$ & $8 \times 10^{23} \frac{\mathrm{W}}{\mathrm{m}^{2}}$ & $7 \times 10^{31} \frac{\mathrm{W}}{\mathrm{m}^{2}}$ \\
\hline Peak electric field & $\mathcal{E}=\sqrt{\mu_{0} c S}$ & $4 \times 10^{14} \frac{\mathrm{V}}{\mathrm{m}}$ & $1 \times 10^{11} \frac{\mathrm{V}}{\mathrm{m}}$ & $2 \times 10^{13} \frac{\mathrm{V}}{\mathrm{m}}$ & $2 \times 10^{17} \frac{\mathrm{V}}{\mathrm{m}}$ \\
\hline Peak electric field/critical field & $\mathcal{E} / \mathcal{E}_{c}$ & $3 \times 10^{-4}$ & $1 \times 10^{-7}$ & $1 \times 10^{-5}$ & 0.1 \\
\hline Photon energy/e rest energy & $\frac{\hbar \omega}{m_{e} c^{2}}$ & $2 \times 10^{-6}$ & 0.006 & 0.006 & 0.02 \\
\hline Adiabaticity parameter & $\eta=\frac{\hbar \omega}{e \mathcal{E} \lambda_{e}}$ & $9 \times 10^{-3}$ & $6 \times 10^{4}$ & $5 \times 10^{2}$ & 0.1 \\
\hline
\end{tabular}

difficulty, in the early 1970's the question was raisedf whether intense optical lasers could be employed to study the Schwinger mechanism 24.25. Yet, it was found that all available and conceivable optical lasers did not have enough power density to allow for a sizeable pair creation rate $24,25,26,27,28,29,30,31,32,33,34$.

With the possibility of X-ray lasers at the horizon, this question has been addressed recently again 56.6.6. As a quasi-realistic picture of the electromagnetic field of a laser, a pure electric field oscillating with a frequency $\omega=2 \pi c / \lambda$ was consideredf, under the assumption that the field amplitude $\mathcal{E}$ is much smaller than the Schwinger critical field, and the photon energy is much smaller than the rest energy of the electron,

$$
\mathcal{E} \ll \mathcal{E}_{c}, \quad \hbar \omega \ll m_{e} c^{2} ;
$$

conditions which are well satisfied at realistic X-ray lasers (c. f. Table 2). Under these conditions, it is possible to compute the rate of $e^{+} e^{-}$pair production in a semiclassical manner, using generalized WKB or imaginary-time methods 25.26.28,30.33 34. Here, the ratio $\eta$ of the energy of the laser photons over the work of the field on a unit charge $e$ over the Compton wavelength of the electron,

$$
\eta=\frac{\hbar \omega}{e \mathcal{E} \lambda_{e}}=\frac{\hbar \omega}{m_{e} c^{2}} \frac{\mathcal{E}_{c}}{\mathcal{E}}=\frac{m_{e} c \omega}{e \mathcal{E}}
$$

plays the role of an adiabaticity parameter. As long as $\eta \ll 1$, i. e. in the strong-field, lowfrequency limit, the non-perturbative Schwinger result (8) for a static uniform field applies. On

\footnotetext{
${ }^{b}$ At about the same time, the thorough investigation of the question started whether the necessary superstrong fields around $\mathcal{E}_{c}$ can be qenerated microscopically and transiently in the Coulomb field of colliding heavy ions with $Z_{1}+Z_{2}>Z_{c} \approx 17022$. At the present time, clear experimental signals for spontaneous positron creation in heavy ion collisions are still missing and could only be expected from collisions with a prolonged lifetime 23 .

${ }^{c}$ Such a field may be created in an antinode of the standing wave produced by a superposition of two laser beams. In Ref. 34 , pair creation in the overlap region of two lasers, whose beams make a fixed angle to each other, was considered.
} 


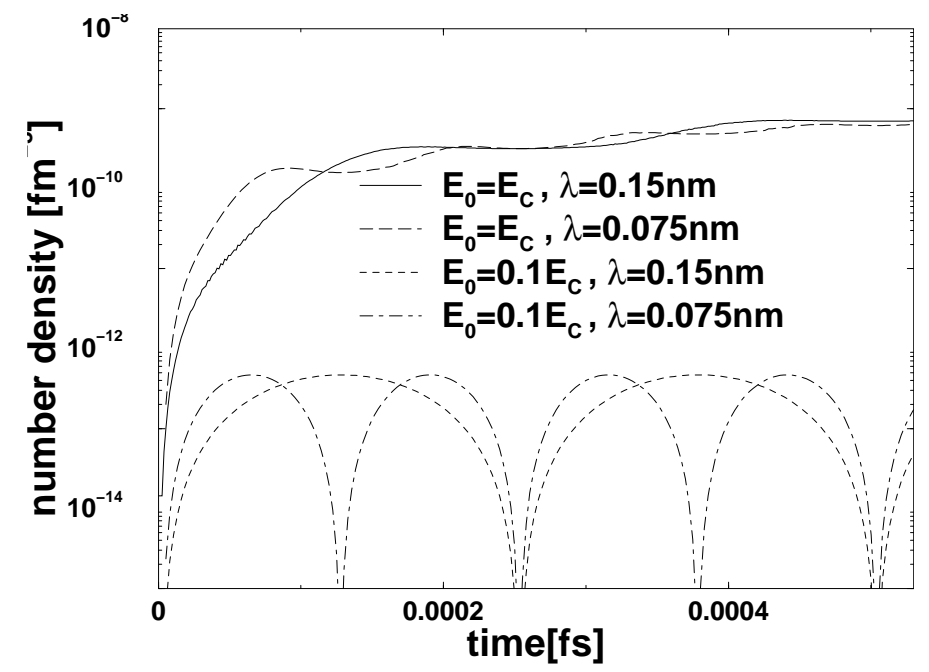

Figure 3: Time evolution of the number density of produced $e^{+} e^{-}$pairs at the focus of an X-ray laser 8 .

the other hand, for large $\eta$, i. e. in the low-field, high-frequency limit, the essentially perturbative result

$$
\frac{\mathrm{d}^{4} n_{e^{+} e^{-}}}{\mathrm{d}^{3} x \mathrm{~d} t} \sim \frac{c}{4 \pi^{3} \lambda_{e}{ }^{4}}\left(\frac{\mathrm{e}}{4} \frac{e E \lambda_{e}}{\hbar \omega}\right)^{4 m_{e} c^{2} / \hbar \omega}
$$

is obtained for the rate of pair production. It corresponds to the $n$-th order perturbation theory, $n$ being the minimum number of quanta required to create an $e^{+} e^{-}$pair: $n \gtrsim 2 m_{e} c^{2} /(\hbar \omega) \gg 1$.

For an X-ray laser, with $\hbar \omega \sim 1 \div 10 \mathrm{keV}$, the adiabatic, nonperturbative, strong field regime, $\eta \lesssim 1$, starts to apply for $\mathcal{E} \gtrsim \hbar \omega \mathcal{E}_{c} /\left(m_{e} c^{2}\right) \sim 10^{15 \div 16} \mathrm{~V} / \mathrm{m}$ (c.f. Eq. (10)). An inspection of the tunneling rate (8) leads then to the conclusion that one needs an electric field of about $0.1 \mathcal{E}_{c} \sim 10^{17} \mathrm{~V} / \mathrm{m}$ in order to get an appreciable amount of spontaneously produced $e^{+} e^{-}$pairs at an X-ray laser $\mathrm{U}$. Under such conditions the production rate is time-dependent, with repeated cycles of particle production and annihilation in tune with the laser frequency (cf. Fig. 觗, but the peak particle number is independent of the laser frequency: up to $10^{3}$ pairs may be produced in the spot volume $\mathrm{B}$.

In Table 2 we have summarized the relevant parameters for the planned X-ray FELs. We conclude that the power densities and electric fields which can be reached with presently available technique (column labeled "Focused: Available" in Table 2) are far too small for a sizeable effect. On the other hand, if the energy extraction can be improved considerably, such that the power of the planned X-ray FELs can be increased to the terawatt region, and if X-ray optics can be improved 37 to approach the diffraction limit of focusing, leading to a spot size in the 0.1 nanometer range, then there is ample room (c.f. column labeled "Focus: Goal" in Table 2) for an investigation of the Schwinger pair production mechanism at X-ray FELs.

\subsection{Unruh Effect}

Black hole evaporation, the so-called Hawking effect 18 , and the similar Unruh effect 18 are two fundamental effects in present-day theoretical physics. Both are thermal-like effects involving microscopic degrees of freedom of quantum fields which are not causally connected (event horizons).

Experimental detection of Hawking radiation from real, massive $\left(M_{\mathrm{bh}}\right)$ general-relativistic black holes seems impossible, since the corresponding temperature, as seen by an outside sta- 


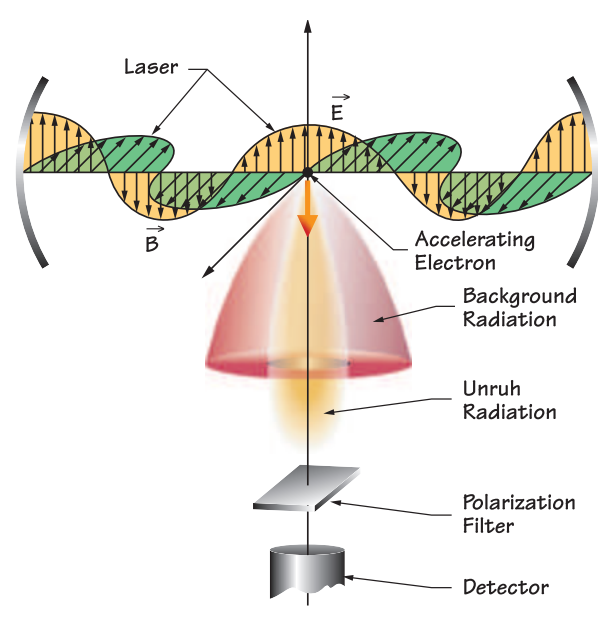

Figure 4: Schematic diagram of an experiment to detect the Unruh effect at an X-ray free electron laser 44.

tionary observer, is tiny,

$$
T_{\text {Hawking }}=\frac{\hbar \kappa}{2 \pi k}=6 \cdot 10^{-8} \mathrm{~K}\left(\frac{1 M_{\odot}}{M_{\mathrm{bh}}}\right) \text {, }
$$

where $\kappa$ is the surface gravity of the black hole, $k$ is the Boltzmann constant, and $M_{\odot}$ denotes the solar mass. Furthermore, the proposed detection 39 of Hawking radiation from primordial mini black holes, which are relics from the big bang, is rather indirect I. Even the detection of condensed matter analogues of Hawking radiation, while more accessible than that from real black holes, is currently far from laboratory realization (see e.g. Ref. 13 and references therein).

Under these circumstances, it seems worthwhile to look more closely onto the Unruh effect. It implies that a particle uniformly accelerated by an acceleration $a$ will find itself surrounded by a thermal heat bath at a temperature

$$
T_{\text {Unruh }}=\frac{\hbar a}{2 \pi c k}=4 \cdot 10^{-21} \mathrm{~K}\left(\frac{a}{1 \mathrm{~m} / \mathrm{s}^{2}}\right) .
$$

We see that enormous accelerations are required to produce a measurable effect. Here, the X-ray lasers come into play: very large accelerations might be available at their focus (cf. Eq. (6)).

A scheme how the Unruh effect could be detected at the focus of an X-ray laser 19 . 11 is shown in Fig. A. In the spot of a standing laser wave, where only an electric field exists, a single electron is accelerated with $a \approx 10^{26} \mathrm{~m} / \mathrm{s}^{2}$, a value which is possible with state-of-the-art means of focusing (cf. Eq. (6) and Table 2, column labeled "Focus: Available"). The acceleration of the electron through the vacuum causes a jitter in the electron's motion, in addition to the usual zero point fluctuations. This jitter modifies the radiation emitted by the electron - over and above the classical Larmor radiation. The additional, acceleration-related radiation has a characteristic $a$ dependence (a distorted thermal spectrum) and angular dependence (cf. Fig. 1). In particular, there is a blind spot in the angular dependence of the classical Larmor radiation. Any radiation in this blind spot should be traceable to the distortion of the zero-point fluctuations.

Whether ultimately one will call this a verification of the Unruh effect or just basic quantum field theory (QED) is a matter of taste or linguistics 11. After all, the Unruh temperature itself will not be measured. Nevertheless, it seems worthwhile to pursue this type of experiment.

\footnotetext{
${ }^{d}$ In models of $\mathrm{TeV}$-scale quantum gravity with extra dimensions 40 , mini black hples may be generated and their evaporation studied at near-future collider 41 and existing cosmic ray facilities 42 .
} 


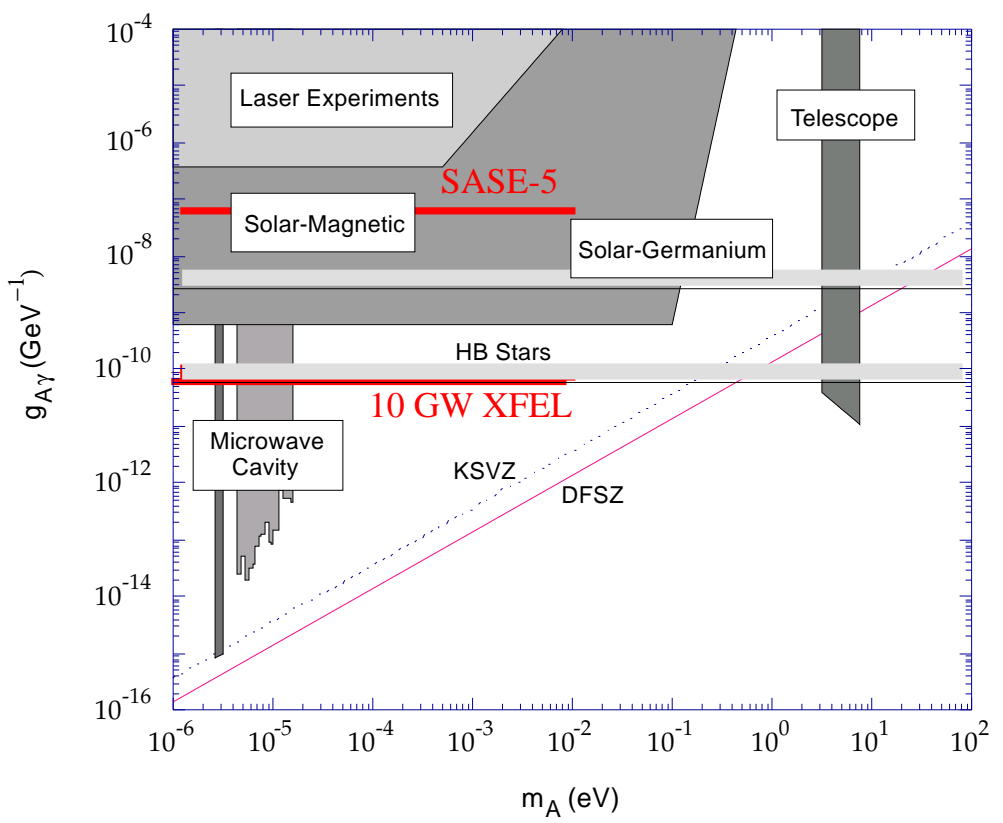

Figure 5: Exclusion region in mass $m_{A}$ vs. axion-photon coupling $g_{A \gamma}$ for various current experiments (adapted from Ref. 47, where also the correspondins references can be found). Also shown in this figure, and labeled with "SASE-5", is the projected sensitivity 13 of a photon regeneration experiment using the SASE-5 XFEL (cf. Table 1), as well as the one of a hypothetical XFEL with average power $\langle P\rangle=10 \mathrm{GW}$ ("10 GW XFEL").

\subsection{Axion Production}

An axion $\left(A^{0}\right) 45$ is a hypothetical, very light, weakly coupled (pseudo-)scalar particle. It arises from a natural solution to the strong $C P$ problem: why the effective $\theta$-parameter in the Lagrangian of quantum chromodynamics (QCD),

$$
\mathcal{L}_{\theta}=\theta_{\text {eff }} \frac{\alpha_{s}}{8 \pi} G^{\mu \nu a} \tilde{G}_{\mu \nu a}
$$

is so small, $\theta_{\text {eff }} \lesssim 10^{-9}$, as required by the current limits on the neutron electric dipole moment, even though $\theta_{\text {eff }} \sim 1$ is perfectly allowed by QCD gauge invariance? Here, $\alpha_{s}$ is the strong fine-structure constant, and $G^{\mu \nu a}\left(\tilde{G}_{\mu \nu a}\right)$ are the (dual) gluon field strength tensors. The axion appears as a pseudo Nambu-Goldstone boson of a spontaneously brocen Peccei-Quinn symmetry 46 , whose scale $f_{A}$ determines the mass,

$$
m_{A}=0.62 \cdot 10^{-3} \mathrm{eV} \times\left(\frac{10^{10} \mathrm{GeV}}{f_{A}}\right) .
$$

The original axion model, with $f_{A} \sim v=247 \mathrm{GeV}$ being of the order of the scale of electroweak symmetry breaking, is experimentally excluded (see e.g. Ref.47 and references therein), however so-called invisible axion models 48.49 , where $f_{A} \gg v$, are still allowed.

The interactions of axions with Standard Model particles are model dependent. Of particular importance is the axion-photon coupling $g_{A \gamma}$,

$$
\mathcal{L}_{\mathrm{WW}}=\frac{1}{4} g_{A \gamma} A^{0} F_{\mu \nu} \tilde{F}^{\mu \nu}=-g_{A \gamma} A^{0} \mathbf{E} \cdot \mathbf{B} ; \quad g_{A \gamma}=\frac{\alpha}{2 \pi f_{A}}\left(\frac{E}{N}-1.92\right)
$$

where $F_{\mu \nu}\left(\tilde{F}^{\mu \nu}\right)$ is the (dual) electromagnetic field strength tensor. The quantity $E / N$ is the ratio of electromagnetic over color anomalies, a model-dependent ratio of order one. It is noteworthy however, that two quite distinct models, namely the so-called KSVZ 48 (or hadronic) 


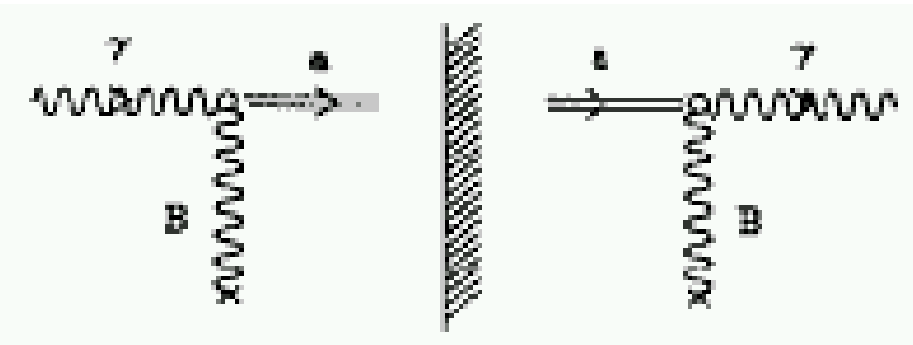

Figure 6: Schematic view of axion production through photon conversion in a magnetic field (left) and subsequent detection through photon regeneration (right) 52.

and the so-called DFSZ49 (or grand unified) one, lead to quite similar axion-photon couplings, as shown in Fig. 5, which displays the axion-photon coupling (16), in terms of its mass (15). Also shown in Fig. 5 are the exclusion regions arising from astrophysical considerations and various experiments. A. Apart from the laser experiments quoted in Fig. 5, all the others rely on axion production in cosmological or astrophysical environments and aim only at their detection: The microwave cavity experiments assume that axions are the galactic dark matter, the telescope search looks for axions thermally produced in galaxy clusters, and the solar-magnetic and solarGermanium experiments search for axions from the sun.

In connection with the X-ray laser, a photon regeneration experiment seems to be appropriate and possible 13 . It may be based on the idea 50 to send a laser beam along a superconducting dipole magnet (with $\mathbf{E} \| \mathbf{B}$ ), in whose magnetic field the photons may convert via a Primakoff process (cf. Eq. (16)) into axions (cf. Fig. 6). If another dipole magnet is set up in line with the first magnet, with a sufficiently thick wall between them, then photons may be regenerated from the pure axion beam in the second magnet and detected. For light axions, with

$$
m_{A} \ll \sqrt{\frac{4 \pi \omega}{\ell}}=1.6 \cdot 10^{-3} \mathrm{eV}\left(\frac{\hbar \omega}{1 \mathrm{eV}} \frac{1 \mathrm{~m}}{\ell}\right)^{1 / 2}
$$

where $\ell$ is the length of the magnetic field, the axion beam produced is collinear and coherent with the photon beam, and the overall rate for production and subsequent detection is

$$
\text { rate } \propto \frac{1}{16}\left(g_{A \gamma} B \ell\right)^{4} \frac{\langle P\rangle}{\omega}
$$

where $\langle P\rangle$ is the average laser power. A pilot experiment 51 52 with an optical laser, $\lambda=514$ $\mathrm{nm}$, of power $\langle P\rangle=3 \mathrm{~W}$, and with a magnet with $B=3.7 \mathrm{~T}$ and $\ell=4.4 \mathrm{~m}$, excluded axion-like pseudo-scalars with mass $m_{A}<10^{-3} \mathrm{eV}$ and axion-photon couplings $g_{A \gamma}>6.7 \cdot 10^{-7} \mathrm{GeV}^{-1}$. The overall envelope of current limits from laser-based experiments is shown in Fig. 5 ("Laser Experiments").

Also shown in this figure, and labeled with "SASE-5", is the projected sensitivity 13 (cf. Eqs. (17) and (18)) of a possible photon regeneration experiment using the SASE-5 XFEL (cf. Table (1), along with a state-of-the-art magnetic field of $B=10 \mathrm{~T}$ over $\ell=10 \mathrm{~m}$. Clearly, in order to reach a sensitivity comparable to the one arising from stellar evolution], which excludes $g_{A \gamma} \gtrsim 6 \cdot 10^{-11} \mathrm{GeV}^{-1}$ and is labeled "HB Stars" in Fig. 5, additional efforts are necessary. For example, one might envisage an XFEL with $\langle P\rangle=10 \mathrm{GW}$, and a magnetic field of $B=40 \mathrm{~T}$ over $\ell=40 \mathrm{~m}$, to obtain a projected sensitivity of $g_{A \gamma} \gtrsim 8 \cdot 10^{-11} \mathrm{GeV}^{-1}$, for $m_{A} \lesssim 10^{-2} \mathrm{eV}$.

\footnotetext{
${ }^{e}$ For a discussion and references, see Ref. 47 .

${ }^{f}$ The Cern Axion Solar Telescope $3(\mathrm{CAST})$ has a designed sensitivity of $g_{A \gamma} \gtrsim 5 \cdot 10^{-11} \mathrm{GeV}^{-1}$ and will compete with the stellar evolution limit.
} 


\section{Conclusions}

We have considered several particle physics applications of X-ray free electron lasers, such as spontaneous $e^{+} e^{-}$pair creation from vacuum, the Unruh effect, and axion production. We have seen that for all these applications still some improvement in X-ray FEL technology over the presently considered design parameters is necessary. But these opportunities appear to be very well worth the effort. In addition, I should point out that the subject of fundamental physics at an X-ray FEL is still in its infancy and in a development phase. No doubt, there will be unpredecedented opportunities to use these intense X-rays in order to explore even more issues of fundamental physics that have eluded man's probing so far.

\section{Acknowledgments}

I would like to thank H. Mais, A. Melissinos, G. Raffelt, and J. Roßbach for fruitful discussions and a careful reading of the manuscript. Furthermore, I would like to congratulate Bill Marciano and Sebastian White for organizing such a remarkable workshop.

\section{References}

1. J. Arthur et al. [LCLS Design Study Group Collaboration], SLAC-R-0521 (1998).

2. R. Brinkmann, G. Materlik, J. Rossbach, and A. Wagner, Hamburg, Germany: DESY (1997) 1183 p. Hamburg DESY - DESY-97-048 (97/05,rec.Sep.) 1183 p. (ECFA 97-182).

3. G. Materlik and T. Tschentscher, TESLA: The superconducting electron positron linear collider with an integrated X-ray laser laboratory, Technical design report, Pt. 5: The Xray free electron laser, DESY-01-011, DESY-2001-011, DESY-01-011E, DESY-2001-011E, DESY-TESLA-2001-23, DESY-TESLA-FEL-2001-05, ECFA-2001-209.

4. T. Tajima, "Fundamental Physics with an X-Ray Free Electron Laser," subm. to Comments Plasma Phys. Contr. Fusion; T. Tajima and G. Mourou, arXiv:physics/0111091.

5. A. C. Melissinos, in Quantum Aspects of Beam Physics, Proc. 15th Advanced ICFA Beam Dynamics Workshop, Monterey, Calif., 4-9 Jan 1998, ed. P. Chen (World Scientific, Singapore, 1998), p. 564.

6. P. Chen and C. Pellegrini, in Quantum Aspects of Beam Physics, Proc. 15th Advanced ICFA Beam Dynamics Workshop, Monterey, Calif., 4-9 Jan 1998, ed. P. Chen (World Scientific, Singapore, 1998), p. 571.

7. A. Ringwald, Phys. Lett. B 510, 107 (2001).

8. R. Alkofer, M. B. Hecht, C. D. Roberts, S. M. Schmidt, and D. V. Vinnik, Phys. Rev. Lett. 87, 193902 (2001).

9. V. S. Popov, Pisma Zh. Eksp. Teor. Fiz. 74, 151 (2001) [JETP Lett. 74, 133 (2001)].

10. P. Chen and T. Tajima, Phys. Rev. Lett. 83, 265 (1999).

11. H. C. Rosu, Phys. World 10, 21 (1999); M. Visser, Matters Grav. 17, 4 (2001) grqc/0102044.

12. A. C. Melissinos, Nucl. Phys. Proc. Suppl. 72, 195 (1999).

13. A. Ringwald, in preparation.

14. J. M. Madey, J. Appl. Phys. 42, 1906 (1971).

15. A. M. Kondratenko and E. L. Saldin, Part. Accel. 10, 207 (1980); R. Bonifacio, C. Pellegrini, and L. M. Narducci, Opt. Commun. 50, 373 (1984).

16. J. Andruszkow et al. [TESLA Collaboration], Phys. Rev. Lett. 85, 3825 (2000).

17. F. Sauter, Z. Phys. 69, 742 (1931); W. Heisenberg and H. Euler, Z. Phys. 98, 714 (1936); J. Schwinger, Phys. Rev. 82, 664 (1951). 
18. S. W. Hawking, Nature 248, 30 (1974); Commun. Math. Phys. 43, 199 (1975); T. Damour and R. Ruffini, Phys. Rev. D 14, 332 (1976); G. W. Gibbons and M. J. Perry, Proc. Roy. Soc. Lond. A 358, 467 (1978); S. P. Gavrilov and D. M. Gitman, Phys. Rev. D 53, 7162 (1996); M. K. Parikh and F. Wilczek, Phys. Rev. Lett. 85, 5042 (2000).

19. A. Casher, H. Neuberger, and S. Nussinov, Phys. Rev. D 20, 179 (1979); B. Andersson, G. Gustafson, G. Ingelman, and T. Sjöstrand, Phys. Rept. 97, 31 (1983); T. S. Biro, H. B. Nielsen, and J. Knoll, Nucl. Phys. B 245, 449 (1984).

20. L. Parker, Phys. Rev. 183, 1057 (1969); N. D. Birrell and P. C. Davies, Quantum Fields in Curved Space (Cambridge University Press, 1982).

21. W. Greiner, B. Müller, and J. Rafelski, Quantum Electrodynamics of Strong Fields (Springer-Verlag, Berlin, 1985); A. A. Grib, S. G. Mamaev, and V. M. Mostepanenko, Vacuum Quantum Effects in Strong Fields (Atomizdat, Moscow, 1988; Friedmann Laboratory Publishing, St. Petersburg, 1994); E. S. Fradkin, D. M. Gitman, and Sh. M. Shvartsman, Quantum Electrodynamics with Unstable Vacuum (Springer-Verlag, Berlin, 1991).

22. Ya. B. Zel'dovich and V. S. Popov, Usp. Fiz. Nauk. 105, 403 (1971) [Sov. Phys. Usp. 14, 673 (1972)]; B. Müller, J. Rafelski, and W. Greiner, Z. Phys. 257, 62 (1972); ibid. 257, 183 (1972).

23. W. Greiner and J. Reinhardt, in Quantum Aspects of Beam Physics, Proc. 15th Advanced ICFA Beam Dynamics Workshop, Monterey, Calif., 4-9 Jan 1998, ed. P. Chen (World Scientific, Singapore, 1998), p. 438.

24. F. V. Bunkin and I. I. Tugov, Dokl. Akad. Nauk Ser. Fiz. 187, 541 (1969) [Sov. Phys. Dokl. 14, 678 (1970)].

25. E. Brezin and C. Itzykson, Phys. Rev. D 2, 1191 (1970).

26. V. S. Popov, Pisma Zh. Eksp. Teor. Fiz. 13, 261 (1971) [JETP Lett. 13, 185 (1971)]; V. S. Popov, Zh. Eksp. Teor. Fiz. 61, 1334 (1971) [Sov. Phys. JETP 34, 709 (1972)].

27. G. J. Troup and H. S. Perlman, Phys. Rev. D 6, 2299 (1972); V. S. Popov, Zh. Eksp. Teor. Fiz. 62, 1248 (1972) [Sov. Phys. JETP 35, 659 (1972)]; V. S. Popov and M. S. Marinov, Yad. Fiz. 16, 809 (1972) [Sov. J. Nucl. Phys. 16, 449 (1973)]; N. B. Narozhnyi and A. I. Nikishov, Zh. Eksp. Teor. Fiz. 65, 862 (1973) [Sov. Phys. JETP 38, 427 (1974)].

28. V. S. Popov, Pisma Zh. Eksp. Teor. Fiz. 18, 435 (1973) [JETP Lett. 18, 255 (1974)].

29. V. M. Mostepanenko and V. M. Frolov, Yad. Fiz. 19, 885 (1974) [Sov. J. Nucl. Phys. 19, 451 (1974)].

30. V. S. Popov, Yad. Fiz. 19, 1140 (1974) [Sov. J. Nucl. Phys. 19, 584 (1974)].

31. M. S. Marinov and V. S. Popov, Fortsch. Phys. 25, 373 (1977).

32. J. I. Katz, Astrophys. J. Supp. 127, 371 (2000) physics/9806027.

33. G. Dunne and T. Hall, Phys. Rev. D 58, 105022 (1998).

34. H. M. Fried, Y. Gabellini, B. H. McKellar, and J. Avan, Phys. Rev. D 63, 125001 (2001).

35. M. Perry and G. Mourou, Science 264, 917 (1994).

36. W. Graeff, private communication.

37. J. B. Hastings et al., "X-ray Laser Physics", in LCLS - The First Experiments (September 2000).

38. W. G. Unruh, Phys. Rev. D 14, 870 (1976).

39. F. Halzen, E. Zas, J. H. MacGibbon, and T. C. Weekes, Nature 353, 807 (1991).

40. N. Arkani-Hamed, S. Dimopoulos, and G. R. Dvali, Phys. Lett. B 429, 263 (1998); I. Antoniadis, N. Arkani-Hamed, S. Dimopoulos, and G. R. Dvali, Phys. Lett. B 436, 257 (1998); N. Arkani-Hamed, S. Dimopoulos, and G. R. Dvali, Phys. Rev. D 59, 086004 (1999); L. J. Randall and R. Sundrum, Phys. Rev. Lett. 83, 3370 (1999).

41. S. B. Giddings and S. Thomas, hep-ph/0106219; S. Dimopoulos and G. Landsberg, Phys. Rev. Lett. 87, 161602 (2001); S. Hossenfelder, S. Hofmann, M. Bleicher, and H. Stöcker, hep-ph/0109085; K. Cheung, hep-ph/0110163. 
42. J. L. Feng and A. D. Shapere, hep-ph/0109106; L. Anchordoqui and H. Goldberg, hepph/0109242; Y. Uehara, hep-ph/0110382; A. Ringwald and H. Tu, hep-ph/0111042.

43. H. C. Rosu, Int. J. Mod. Phys. D 3, 545 (1994).

44. P. Chen, private communication.

45. S. Weinberg, Phys. Rev. Lett. 40, 223 (1978); F. Wilczek, ibid. 40, 279 (1978).

46. R. D. Peccei and H. R. Quinn, Phys. Rev. Lett. 38, 1440 (1977); Phys. Rev. D 16, 1791 (1977).

47. D. E. Groom et al. [Particle Data Group Collaboration], Eur. Phys. J. C15, 1 (2000).

48. J. E. Kim, Phys. Rev. Lett. 43, 103 (1979); M. A. Shifman, A. I. Vainshtein, and V. I. Zakharov, Nucl. Phys. B166, 493 (1980).

49. A. R. Zhitnitsky, Sov. J. Nucl. Phys. 31, 260 (1980) [Yad. Fiz. 31, 497 (1980)]; M. Dine and W. Fischler, Phys. Lett. B 120, 137 (1983).

50. A. A. Anselm, Yad. Fiz. 42, 1480 (1985); K. Van Bibber, N. R. Dagdeviren, S. E. Koonin, A. Kerman, and H. N. Nelson, Phys. Rev. Lett. 59, 759 (1987).

51. G. Ruoso et al., Z. Phys. C 56, 505 (1992).

52. R. Cameron et al., Phys. Rev. D 47, 3707 (1993).

53. G. Fanourakis et al., CERN-SPSC-2001-020. 